\title{
Factors affecting sufentanil consumption for intravenous controlled analgesia after hepatectomy: retrospective analysis
}

\author{
Yue Wu${ }^{1}$, Lina Tian ${ }^{1}$, Chunye Li ${ }^{2}$, Minjun Liư ${ }^{3}$, Shina Qiao ${ }^{3}$, Weibo Zhang ${ }^{1}$, Suming Tian ${ }^{1}$ and Gang Chen ${ }^{* *}$ (e)
}

\begin{abstract}
Background: Pain control after hepatectomy is usually achieved by opioids. There are significant individual differences in the amount of opioids used after hepatectomy, and the metabolism of opioids is liver-dependent. The purpose of our study was to explore the possible risk factors for opioid consumption during the first $48 \mathrm{~h}$ after surgery.

Methods: In a retrospective study design involving 562 patients undergoing open or laparoscopic hepatectomy, all patients were treated with intravenous patient-controlled analgesia (IV-PCA) along with continuous and bolus doses of sufentanil for a duration of $48 \mathrm{~h}$ after surgery during the time period of August 2015 and February 2019. The primary endpoint was high sufentanil consumption $48 \mathrm{~h}$ after hepatectomy, and patients were divided into two groups: those with or without a high PCA sufentanil dosage depending on the third quartile (Q3). The secondary endpoint was the effect of a high PCA sufentanil dosage on various possible clinical risk factors. The relevant parameters were collected, and correlation and multivariate regression analyses were performed.
\end{abstract}

Results: The median operation time was $185 \mathrm{~min}$ (range, 115-250 min), and the median consumption of sufentanil $48 \mathrm{~h}$ after the operation was $91 \mathrm{\mu g}(\mathrm{IQR}, 64.00,133.00)$. Factors related to the consumption of sufentanil at $48 \mathrm{~h}$ after hepatectomy included age, operation time, blood loss, intraoperative infusion (red blood cells and fresh-frozen plasma), pain during movement after surgery (day 1 and day 2), preoperative albumin, and postoperative blood urea nitrogen. Age ( $\leq 60$ and $>60$ years), extent of resection (minor hepatic resection and major hepatic resection), surgical approach (laparoscope and open) and operation time ( $\mathrm{min}$ ) were independent risk factors for sufentanil consumption at $48 \mathrm{~h}$ postoperatively.

Conclusion: Age younger than 60 years, major hepatic resection, an open approach and a longer operation are factors more likely to cause patients to require higher doses of sufentanil after hepatectomy, and the early identification of such patients can increase the efficacy of perioperative pain management.

Keywords: Influencing factors, Postoperative pain, Patient-controlled analgesia, Sufentanil consumption, Hepatectomy

\footnotetext{
${ }^{*}$ Correspondence: chengang120@zju.edu.cn

'Department of Anesthesiology, Sir Run Run Shaw Hospital, School

of Medicine, Zhejang University, 3 Qingchun Road East, ShangCheng District, Hangzhou 310016 Zhejiang, People's Republic of China

Full list of author information is available at the end of the article
}

\begin{abstract}
Background
Severe pain stress occurs after liver cancer surgery, which may lead to severe liver function damage, increase immune suppression, and promote postoperative infection, tumor recurrence and metastasis $[1,2]$. Continuous and adequate analgesia can reduce postoperative pain, decrease postoperative complications, and improve postoperative patient satisfaction. Additionally, analgesia
\end{abstract}

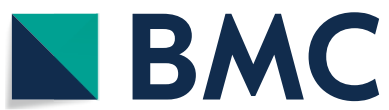

(c) The Author(s) 2021. Open Access This article is licensed under a Creative Commons Attribution 4.0 International License, which permits use, sharing, adaptation, distribution and reproduction in any medium or format, as long as you give appropriate credit to the original author(s) and the source, provide a link to the Creative Commons licence, and indicate if changes were made. The images or other third party material in this article are included in the article's Creative Commons licence, unless indicated otherwise in a credit line to the material. If material is not included in the article's Creative Commons licence and your intended use is not permitted by statutory regulation or exceeds the permitted use, you will need to obtain permission directly from the copyright holder. To view a copy of this licence, visit http://creativecommons.org/licenses/by/4.0/. The Creative Commons Public Domain Dedication waiver (http://creativeco mmons.org/publicdomain/zero/1.0/) applies to the data made available in this article, unless otherwise stated in a credit line to the data. 
plays a crucial role in improving perioperative safety, for example, enhancing the protection of immune function, reducing postoperative infection and promoting the recovery of liver function [3-5]. Therefore, adequate postoperative analgesia is crucial to improve the prognosis of patients undergoing liver cancer surgery.

Laparoscopic hepatectomy has been widely used and carried out in hospitals worldwide. Although it is a minimally invasive surgery with a small incision, laparoscopic surgery can also cause severe visceral pain in deep tissues due to the unique anatomical structure of the liver and the unusually rich liver blood vessels. Moreover, carbon dioxide pneumoperitoneum stimulates the gut and somatic nerves [6], which can also aggravate surgical trauma and pain stress.

Therefore, pain not only after open hepatectomy but also after laparoscopic surgery should be of great concern. Multimode analgesia is an important concept and method of postoperative analgesia, which requires the combination of analgesic drugs with different mechanisms of action and different analgesic measures to achieve the best analgesic effect and minimize adverse reactions. Opioid drugs are indispensable in postoperative analgesia, and patient-controlled analgesia (PCA) is the most commonly used and ideal postoperative analgesia method, especially for abdominal surgery. An increasing number of studies have proven that sufentanil, as a commonly used opioid, has significant advantages in the safety and effectiveness of postoperative intravenous PCA (IV-PCA) [7]. However, there are substantial individual differences in opioid use. The characteristics of the patient, tumor, surgery, and even anesthesia may influence the amount of opioids used after surgery. The metabolism and elimination of opioids depends on liver and kidney function. Other studies have shown that preoperative platelet and coagulation abnormalities are independent risk factors for significant postoperative mortality in patients with liver cancer [8] and may also have an impact on postoperative opioid use. Therefore, the individualized postoperative PCA scheme can help to reduce postoperative pain stress, improve patient satisfaction, and reduce the waste of workforce and material resources.

The early identification of risk factors for postoperative opioid use and increased awareness of the importance of related risk factors will contribute to more effective intervention and better pain management. In this study, patients with primary liver cancer who underwent laparoscopic or open hepatectomy and received IV-PCA after surgery were selected as the study subjects. We retrospectively analyzed the influencing factors of the postoperative sufentanil dosage to provide a basis for individualized postoperative analgesic treatment.

\section{Methods}

The Institutional Review Board of Sir Run Run Shaw Hospital of Zhejiang University, Hangzhou, Zhejiang, P.R. China approved the study protocol. A retrospective review of all patients undergoing hepatectomy at a single institution between August 2015 and February 2019 was performed. All patients with primary liver cancer who underwent hepatectomy were included in the study. All cases were confirmed to be hepatocellular carcinoma by postoperative histopathology. The exclusion criteria were (1) a history of chronic pain and mental illness, (2) the use of analgesics before surgery, (3) transfer to the intensive care unit (ICU) after surgery, and (4) the use of opioids other than sufentanil postoperatively.

Types of hepatic resection were defined by consensus [9]. Right hepatectomy, left hepatectomy, extended right hepatectomy, and extended left hepatectomy were considered major hepatic resection (three segments or more), whereas segmentectomy of one or two segments and nonanatomic wedge resection were classified as minor hepatic resection (two segments or fewer).

All patients undergoing either open or laparoscopic hepatectomy received general anesthesia with endotracheal intubation. Anesthesia was induced by sufentanil (IDT Biologika GmbH, Ampharmapark D-06861 DessauRoblau, Germany), propofol (Fresenius Kabi Deutschland $\mathrm{GmbH}$, Germany), benzocisuatracurium (Zhejiang Xianju Pharmaceutical Co., Ltd., China) or rocuronium bromide (Zhejiang Xianju Pharmaceutical Co., Ltd., China), and anesthesia was maintained by the continuous infusion of remifentanil (Yichang Renfu Pharmaceutical Co., Ltd., China) and inhalation of sevoflurane (Shanghai Hengrui Pharmaceutical Co., Ltd., China) and intermittent injection of benzocisuatracurium or rocuronium bromide.

According to the patient's condition and the preference of the anesthesiologist, most patients were intravenously infused with dexmedetomidine (Yangzijiang Pharmaceutical Group Co., Ltd., China) during the operation. At the end of laparoscopic surgery, $10-15 \mathrm{ml}$ of $0.375 \%$ ropivacaine (AstraZeneca AB, Britain) was routinely administered for incisional local infiltration analgesia.

The IV-PCA regimen typically consisted of sufentanil $250 \mu \mathrm{g}$ plus normal saline (total volume of $250 \mathrm{ml}$ ). Sufentanil was administered through a pump, programmed to deliver $1 \mathrm{ml} /$ hour as a background infusion, and $2 \mathrm{ml}$ per demand. Postoperative pain at rest and during movement, such as coughing and turning over, was recorded and assessed using a numerical rating scale (NRS) [10] ranging from 0 , indicating no pain, to 10 , indicating severe pain. The patient selected the corresponding number to indicate the degree of pain, with an NRS score $\leq 3$ serving as the control target. Preoperative 
patients routinely received education on how to use the PCA pump. If the postoperative NRS score was $\geq 4$, they could give the required amount (bolus dose) by themselves through the button until they reached an NRS score $\leq 3$. The nurse in charge performed a routine pain assessment once every $4 \mathrm{~h}$; an intervention and reassessment were given when the NRS score was $\geq 4$, and routine assessment was resumed when the NRS score was $\leq 3$. In addition, the Acute Pain Service Team (APS) conducted postoperative follow-up examinations twice a day for patients who used a PCA pump after hepatectomy to observe and record the patient's pain score and adverse reactions, such as pain at rest or during movement that could not be effectively controlled or medication overdoses causing drowsiness and even respiratory depression, to adjust the analgesic pump parameters in a timely manner. At other times, if the doctor in charge or nurse reported the patient's pain or adverse reaction in a timely manner, the APS team addressed it as soon as possible.

In addition to sufentanil, many patients received a routine intravenous injection of anti-inflammatory and analgesic drugs (nonsteroidal anti-inflammatory drugs (NSAIDs)), such as flurbiprofen (Beijing Tide Pharmaceutical Co., Ltd., China) or parecoxib (Pharmacia \& Upjohn Company LLC, USA), in the first $48 \mathrm{~h}$ after surgery.

Patients were routinely transferred to the general ward after hepatectomy (which differed from practice at other hospitals) unless there were issues such as respiratory and circulatory instability, unusual surgical complexity, and excessive bleeding. Routine blood and biochemical tests were performed within $48 \mathrm{~h}$ after surgery. Postoperative nausea and vomiting (PONV) are usually treated with metoclopramide (Suicheng Pharmaceutical Co., Ltd., China) or ondansetron (Qilu Pharmaceutical Co., Ltd., China).

Patient demographics and clinical data from the medical records included sex, age, body mass index (BMI; weight $(\mathrm{kg}) /$ height (meters) squared), tumor factors (tumor size, tumor number, differentiation, vascular invasion and lymph node invasion), perioperative factors (American Society of Anesthesiologists (ASA) physical status, surgical approach, extent of resection, operation time, cut margin, intraoperative blood loss, intraoperative transfusion, intraoperative dexmedetomidine use, postoperative NSAID use, postoperative NRS score, postoperative sufentanil dosage and postoperative adverse reactions) and laboratory data (platelet count, prothrombin time, indicators of liver and kidney function).

The primary endpoint was the level of sufentanil consumption $48 \mathrm{~h}$ after hepatectomy and the third quartile (Q3, $133 \mu \mathrm{g})$. The patients were divided into two groups: those with or without a high PCA sufentanil dosage depending on this value. Other parameters were compared between groups to assess whether there were differences. The secondary endpoint was the effect of a high PCA sufentanil dosage on various possible clinical risk factors, for example, patient demographics and clinical data, including sex, age, BMI, tumor factors, perioperative factors and laboratory data.

\section{Statistical analysis}

All continuous data with a normal distribution are described as the mean $\pm S D$, and continuous data with a nonnormal distribution are described as the median and interquartile range. Normality was assessed using the Shapiro-Wilk test. Categorical variables are expressed as numbers and percentages. Univariate analysis was performed on the preoperative demographic data and perioperative general characteristics of patients undergoing liver resection, including age, sex, BMI, intraoperative blood loss, operating time, intraoperative transfusion, extent of resection, surgical approach, ASA classification, intraoperative DEX, postoperative NSAIDs, PONV, pain score, sufentanil dosage, laboratory data and tumorrelated factors, and independent sample t-test or MannWhitney $U$ test were used to compare the differences between the PCA-NH and PCA-H groups; and the independent sample t-test or Mann-Whitney $U$ test was also used to compare the difference in sufentanil consumption in PCA for each category. For categorical parameters, chi-square analysis and Fisher's exact test were used when appropriate. Correlation analysis between possible risk factors and sufentanil consumption was carried out. The related variables and the factors that may affect the postoperative sufentanil dosage obtained from the univariate analysis were included in the multivariate regression analysis to identify the risk factors for postoperative PCA sufentanil consumption. Data were considered statistically significant at a $P$-value of less than 0.05 . All analyses were performed using SPSS Statistics software (Version 23.0, Chicago, IL, USA).

\section{Results}

A total of 839 patients at the Sir Run Run Shaw Hospital underwent hepatectomy by a laparoscopic or open approach between August 2015 and February 2019. Of these, 54 were excluded due to preoperative chronic opioid use, 53 were excluded due to transfer to the ICU postoperatively, 102 were excluded due to missing or incomplete medical records, and 68 were excluded due to emergency surgery. As a result, 562 patients were included in the final analysis. A flowchart of the patient selection process is shown in Fig. 1. 


\section{9 liver cancer patients underwent hepatectomy}

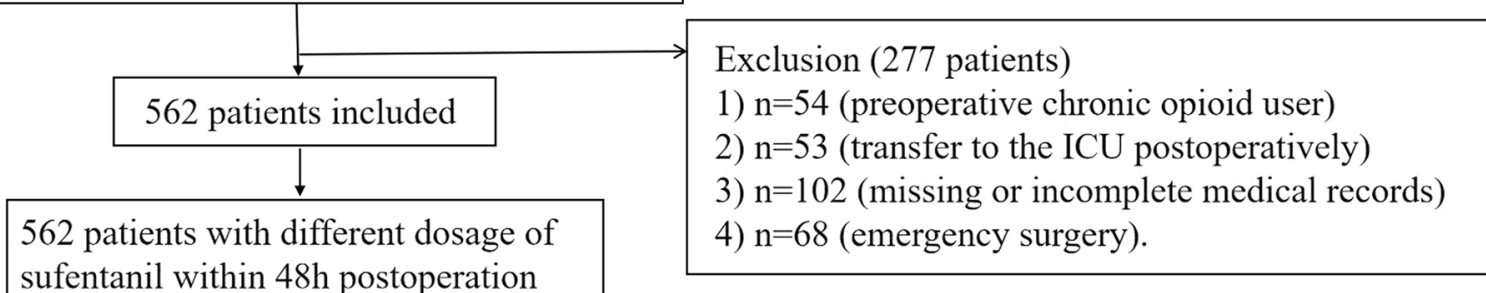

Patient demographics and clinical data included: gender, age, BMI, tumor factors (tumor size, tumor number, differentiation, vascular invasion and lymph node invasion), perioperative factors (ASA physical status, surgical approach, extent of resection, operative time, cut margin, intraoperative blood loss, intraoperative transfusion, intraoperative dexmedetomidine use, postoperative NSAIDs use, postoperative NRS, postoperative sufentanil dosage and postoperative adverse reactions) and laboratory data (platelet count, prothrombin time, liver and kidney function).

Fig. 1 Patient selection flowchart

\section{Patient characteristics and perioperative variables}

The preoperative demographic data and general perioperative characteristics of the total patient sample and the two groups of patients (with and without a high PCA sufentanil dosage, i.e., the PCA-NH and PCA-H groups) are presented in Table 1 . Among the 562 participants, there were slightly more males $(316,56.2 \%)$ than females. The median age was 57.0 years (range, $18 \sim 88 \mathrm{ml}$ ), and the median BMI was $22.7 \mathrm{~kg} / \mathrm{m}^{2}$ (range, $15.38 \sim 33.87 \mathrm{~kg} / \mathrm{m}^{2}$ ). Pain during movement was significantly higher than pain at rest 2 days after the operation $(P<0.05)$, and the scores of pain at rest and during movement on the second day after the operation were significantly lower than those on the first day $(P<0.05)$ (Fig. 2). Thirty-seven patients (6.6\%) had nausea and vomiting, 25 (4.4\%) had dizziness, and $29(5.2 \%)$ had drowsiness $48 \mathrm{~h}$ after hepatectomy, but no life-threatening opioid-related side effects (such as respiratory depression) were observed.

There was no significant difference in BMI, intraoperative infusion of red blood cells (RBCs), ASA classification, intraoperative dexmedetomidine use, postoperative NSAID use, PONV, or pain scores 2 days after surgery. Compared with the PCA-NH group, the PCA-H group included younger patients, more males, more intraoperative transfusions, more major hepatic resections, and more open approaches $(P<0.05)$.

Table 2 shows the types of hepatic resection performed. Major hepatic resection was performed in 290 cases
(51.6\%), segmentectomy in 147 cases (26.2\%), and localized resection in 125 cases (22.2\%). All operations were mainly performed by open and laparoscopic approaches, including 260 cases of open surgery (46.3\%) and 302 cases of laparoscopic surgery (53.7\%), with a median surgical duration of $185 \mathrm{~min}$ (range, $115 \sim 250 \mathrm{~min}$ ). The median intraoperative blood loss was $200 \mathrm{ml}$ (range, $50 \sim 8000 \mathrm{ml}$ ), and intraoperative transfusion consisted mainly of RBCs and fresh-frozen plasma (FFP). Blood transfusion was required in 198 patients (35.2\%), with a median of $0 \mathrm{U}$ (range, $0 \sim 12.5 \mathrm{U}$ ) of RBCs and $0 \mathrm{ml}$ (range, $0 \sim 3780 \mathrm{ml}$ ) of FFP (Table 1).

The preoperative Child-Pugh grading of liver function in all patients was grade A. Table 3 shows the perioperative laboratory data of the total patient sample and the two groups of patients, including the platelet count, prothrombin time, and indicators of liver and kidney function (alanine aminotransferase, aspartate aminotransferase, alkaline phosphatase, albumin, urea nitrogen and creatinine). There were statistically significant differences in the preoperative BUN level, preoperative creatinine level, preoperative platelet count, and postoperative BUN level between the two groups. There were no significant differences in the other laboratory data between the two groups. The results are described in Table 3.

The results related to tumor factors were obtained from pathological reports. The median tumor size was $3.2 \mathrm{~cm}$ 
Table 1 The preoperative demographic data and general perioperative characteristics of patients undergoing hepatectomy

\begin{tabular}{|c|c|c|c|c|c|c|}
\hline & \multirow{2}{*}{$\begin{array}{l}\text { All ( } n=562) \\
\text { Median (IQR) }\end{array}$} & \multicolumn{2}{|c|}{ PCA-NH $(n=428)$} & \multicolumn{2}{|c|}{ PCA-H $(n=134)$} & \multirow[t]{2}{*}{$P$-value } \\
\hline & & n (\%) & Median (IQR) & n (\%) & Median (IQR) & \\
\hline Age (years old) & $57.00(48.00,65.00)$ & & $59.00(49.00,65.00)$ & & $53.78(44.83,61.58)$ & $0.002^{*}$ \\
\hline \multicolumn{7}{|l|}{ Gender } \\
\hline Male & & $230(72.8)$ & & $86(27.2)$ & & $0.034^{*}$ \\
\hline Female & & $198(80.5)$ & & $48(19.5)$ & & \\
\hline BMI (kg/m2) & $22.7(20.8,22.3)$ & & $22.60(20.67,25.16)$ & & $23.35(21.22,25.30)$ & 0.307 \\
\hline Intraoperative blood loss (ml) & $200(100,500)$ & & $200.00(100.00,500.00)$ & & $292.31(141.67,714.29)$ & $0.001^{*}$ \\
\hline Operative time (min) & $210(160,300)$ & & $205(156.25,285.00)$ & & $245.38(182.08,320.00)$ & $0.002^{*}$ \\
\hline \multicolumn{7}{|l|}{ Intraoperative transfusion } \\
\hline No & & $289(79.4)$ & & 75 (20.6) & & $0.015^{*}$ \\
\hline Yes & & $139(70.2)$ & & $59(29.8)$ & & \\
\hline $\mathrm{RBC}(\mathrm{u})$ & $0(0,2.00)$ & & $0(0,1.50)$ & & $0.73(0,2.11)$ & 0.164 \\
\hline $\mathrm{FFP}(\mathrm{ml})$ & $0(0,352.50)$ & & $0(0,315.00)$ & & $1.42(0,385.71)$ & $0.034^{*}$ \\
\hline \multicolumn{7}{|l|}{ Extent of resection } \\
\hline Minor hepatic resection & & $246(90.4)$ & & $26(9.6)$ & & $<0.001^{*}$ \\
\hline Major hepatic resection & & $182(62.8)$ & & $108(37.2)$ & & \\
\hline \multicolumn{7}{|l|}{ Surgical approach } \\
\hline Laparoscope & & $251(83.1)$ & & $51(16.9)$ & & $<0.001^{*}$ \\
\hline Open & & $177(68.1)$ & & $83(31.9)$ & & \\
\hline \multicolumn{7}{|l|}{ ASA classification } \\
\hline $1+\|$ & & $386(76.3)$ & & $120(23.7)$ & & 0.831 \\
\hline III & & $42(75.0)$ & & $14(25.0)$ & & \\
\hline \multicolumn{7}{|l|}{ Introperative DEX } \\
\hline No & & $52(77.6)$ & & $15(22.4)$ & & 0.766 \\
\hline Yes & & $376(76.0)$ & & $119(24.0)$ & & \\
\hline \multicolumn{7}{|l|}{ Postoperative NSAIDs } \\
\hline No & & $301(76.8)$ & & $91(23.2)$ & & 0.595 \\
\hline Yes & & $127(74.7)$ & & $43(25.3)$ & & \\
\hline \multicolumn{7}{|l|}{ PONV } \\
\hline No & & $404(77.0)$ & & $121(23.0)$ & & 0.095 \\
\hline Yes & & $24(64.9)$ & & $13(35.1)$ & & \\
\hline Resting pain score on POD1 & $2(0,3)$ & & $2(0.25,3)$ & & $2(0,3)$ & 0.251 \\
\hline Movement pain score on POD1 & $5(4,6)$ & & $5(4,6)$ & & $5(5,6)$ & 0.503 \\
\hline Resting pain score on POD2 & $1(0,2)$ & & $1(0,2)$ & & $1(0,2)$ & 0.823 \\
\hline Movement pain score on POD2 & $3(3,4)$ & & $3(3,4)$ & & $4(3,4)$ & 0.056 \\
\hline Sufentanil dosage $24 \mathrm{~h}(\mu \mathrm{g})$ & $50(34,75)$ & & $42.00(31.00,56.75)$ & & $98.00(80.00,118.00)$ & $<0.001^{*}$ \\
\hline Sufentanil dosage $48 \mathrm{~h}(\mu \mathrm{g})$ & $91(64,133)$ & & $77.00(60.00,101.75)$ & & $173.00(149.75,205.75)$ & $<0.001^{*}$ \\
\hline
\end{tabular}

*P value $<0.05$ statistically significant, whether it is a high PCA sufentanil dose comparison between the two groups; Mann-Whitney $U$ test; Chi-square test; $\mathrm{n}(\%)=$ number (percentage); PCA-H High PCA sufentanil dosage, PCA-NH Non high PCA sufentanil dosage, DEX Dexmedetomidine, NSAIDs Anti-inflammatory and analgesic drugs, $R B C(u)$ Intraoperative transfusion of red blood cells (unit), FFP $(\mathrm{ml})$ Intraoperative transfusion of fresh frozen plasma ( $\mathrm{ml}$ ), POD1 Postoperative first day, POD2 Postoperative second day

(range, $0.3-18 \mathrm{~cm}$ ). There were 71 cases of multiple tumors (12.6\%), 138 cases of poor differentiation $(24.6 \%)$, 60 cases of vascular infiltration (10.7\%) and 116 cases of lymph node infiltration (20.6\%). Table 4 shows a comparison of tumor factors between the two groups. There were no statistically significant differences in tumor factors between the two groups, including the tumor number, tumor size, cut margin, differentiation, vascular invasion and lymph node infiltration.

\section{Sufentanil consumption $48 \mathrm{~h}$ after hepatectomy}

The dosage of sufentanil at $48 \mathrm{~h}$ after hepatectomy varied widely, with the quartile range of consumption ranging from 64 to $133 \mu \mathrm{g}$. The median sufentanil 


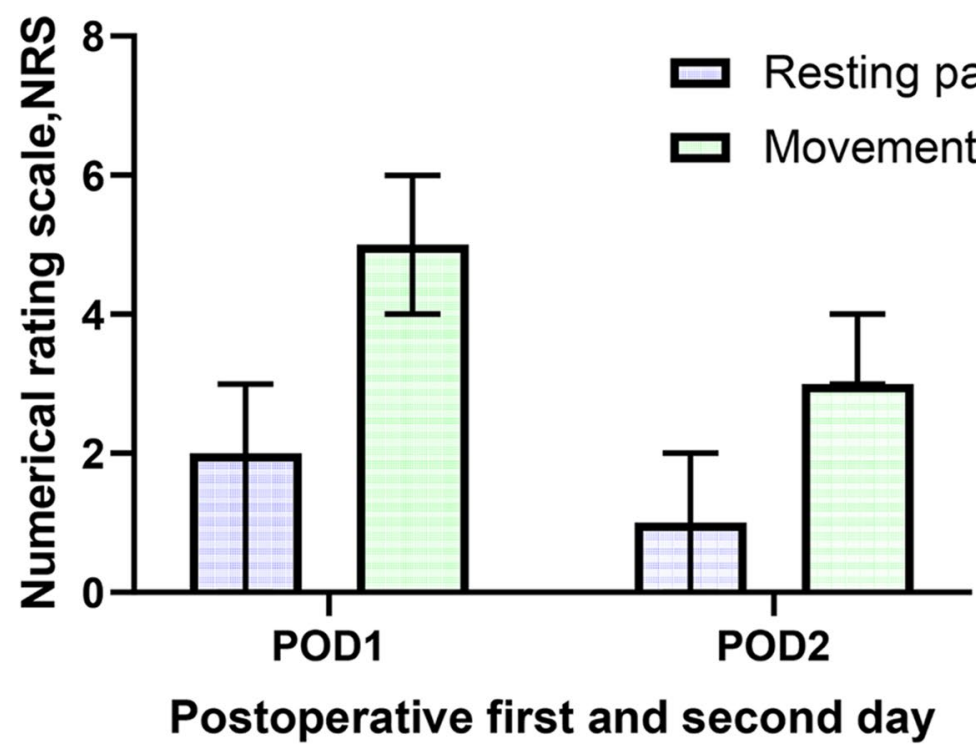

Fig. 2 Postoperative pain score on day 1 and day 2 (median, IQR)

Table 2 Types of hepatic resection

\begin{tabular}{ll}
\hline Type of resection & N (\%) \\
\hline Major hepatic resection & $290(51.6)$ \\
Right lobectomy & $159(28.3)$ \\
Right hepatectomy & $87(15.5)$ \\
Left hepatectomy & $44(7.8)$ \\
Segmentectomy & $147(26.2)$ \\
Left lateral segmentectomy & $75(13.3)$ \\
Typical segmentectomy & $72(12.8)$ \\
Localized resection & $125(22.2)$ \\
\hline
\end{tabular}

consumption on the second day after surgery was less than twice as high as that on the first day, which may have been associated with less pain on the second day after surgery and less need for opioids.

After grouping the different factors, the amount of sufentanil at $48 \mathrm{~h}$ postoperatively was compared between the groups by the Mann-Whitney U-test, and it was found that the consumption of sufentanil was higher in cases of age $\leq 60$ years $(P<0.001)$, major hepatic resection $(P<0.001)$, open approach $(P<0.001)$, operation time $>300 \mathrm{~min}(P=0.008)$, intraoperative transfusion $(P=0.002)$ and PONV $(P=0.030)$. Sex, BMI, ASA classification, intraoperative blood loss, intraoperative infusion of RBCs, intraoperative dexmedetomidine use, postoperative NSAID use, and other indicators showed no significant differences between the groups $(P>0.05)$ (Table 5$)$.

\section{Correlation analysis of sufentanil consumption $48 \mathrm{~h}$ after surgery}

In this study, as the variables did not conform to a normal distribution, Spearman correlation analysis was used to determine the relationship between sufentanil consumption $48 \mathrm{~h}$ postoperatively and other factors, including age, BMI, operation time, blood loss, intraoperative infusion of RBCs and FFP, pain at rest and during movement after surgery (day 1 and day 2), tumor size and laboratory data (including platelet count, prothrombin time, and indicators of liver and kidney function). The results showed that factors related to sufentanil consumption in the first $48 \mathrm{~h}$ after surgery were age, operation time, blood loss, intraoperative infusion of RBCs and FFP, pain during movement after surgery (day 1 and day 2), preoperative albumin and postoperative blood urea nitrogen (BUN), which showed a significant weak correlation $(P<0.05)$ (Table 6$)$. Among them, age was negatively correlated with the increase in sufentanil consumption at $48 \mathrm{~h}$ after surgery; that is, as age decreased, the consumption gradually increased. Other factors were positively correlated with the increase in sufentanil consumption at $48 \mathrm{~h}$ after surgery; as these factors, including operation time, blood loss, intraoperative infusion of RBCs and FFP, movement pain after surgery (day 1 and day 2), preoperative albumin and postoperative BUN, increased, the consumption gradually increased.

\section{Risk factors for sufentanil use $\mathbf{4 8} \mathrm{h}$ after hepatectomy}

The variables included in the multivariate analysis were based on the results of the univariate and 
Table 3 Perioperative laboratory data of patients undergoing hepatectomy

\begin{tabular}{|c|c|c|c|c|}
\hline & All $(n=562)$ & PCA-NH $(n=428)$ & PCA-H $(n=134)$ & $P$-value \\
\hline & \multicolumn{4}{|l|}{ Median, IQR } \\
\hline \multicolumn{5}{|l|}{ Preoperative laboratory data } \\
\hline AST (U/I) & $29.50(21.00,49.00)$ & $30.00(21.00,52.75)$ & $28.00(21.00,44.00)$ & 0.204 \\
\hline $\operatorname{ALT}(U / I)$ & $26.00(16.00,45.00)$ & $27.00(16.25,46.00)$ & $25.33(15.38,38.00)$ & 0.179 \\
\hline Alkaline phosphatase (U/l) & $109.00(81.00,181.50)$ & $110.50(83.00,193.25)$ & $106.33(79.33,164.00)$ & 0.159 \\
\hline Albumin $(\mathrm{g} / \mathrm{l})$ & $38.30(34.70,42.43)$ & $38.20(34.60,42.18)$ & $39.03(35.10,43.60)$ & 0.139 \\
\hline $\mathrm{BUN}(\mathrm{mmol} / \mathrm{l})$ & $4.89(3.89,6.01)$ & $4.79(3.78,5.87)$ & $5.18(4.12,6.38)$ & $0.008^{*}$ \\
\hline Creatinine $(\mu \mathrm{mol} / \mathrm{l})$ & $67.00(56.00,80.00)$ & $66.00(55.00,80.00)$ & $70.00(58.63,82.25)$ & $0.047^{*}$ \\
\hline Platelet count $\left(\times 10^{9} / 1\right)$ & $190.00(138.00,242.00)$ & $193.00(142.25,244.50)$ & $177.20(115.40,232.00)$ & $0.043^{*}$ \\
\hline Prothrombin time (s) & $13.10(12.60,13.80)$ & $13.00(12.60,13.70)$ & $13.25(12.59,14.06)$ & 0.158 \\
\hline \multicolumn{5}{|l|}{ Postoperative laboratory data } \\
\hline AST (U/I) & $101.00(44.00,209.00)$ & $97.50(45.00,211.00)$ & $110.50(41.00,208.00)$ & 0.899 \\
\hline $\operatorname{ALT}(\mathrm{U} / \mathrm{l})$ & $84.50(37.00,188.25)$ & $87.00(37.00,186.75)$ & $73.33(30.00,196.67)$ & 0.531 \\
\hline Alkaline phosphatase (U/l) & $96.00(67.00,162.75)$ & $97.50(69.00,170.00)$ & $92.33(63.75,140.00)$ & 0.145 \\
\hline Albumin $(g / l)$ & $33.10(29.60,36.13)$ & $33.10(29.93,36.08)$ & $33.04(28.70,36.40)$ & 0.520 \\
\hline BUN (mmol/l) & $4.38(3.41,5.44)$ & $4.26(3.37,5.27)$ & $4.66(3.63,5.65)$ & $0.014^{*}$ \\
\hline Creatinine $(\mu \mathrm{mol} / \mathrm{l})$ & $66.50(55.00,80.00)$ & $66.00(54.00,79.75)$ & $67.00(56.70,82.89)$ & 0.111 \\
\hline
\end{tabular}

${ }^{*} P$ value $<0.05$ statistically significant, whether it is a high PCA sufentanil dose comparison between the two groups; Mann-Whitney $U$ test; Chi-square test; $\mathrm{n}(\%)=$ number (percentage); $P C A-H$ High PCA sufentanil dosage, PCA-NH Non high PCA sufentanil dosage

Table 4 Comparison of tumor factors between the PCA-NH group and the PCA-H group

\begin{tabular}{|c|c|c|c|c|c|}
\hline & \multicolumn{2}{|c|}{ PCA-NH $(n=428)$} & \multicolumn{2}{|c|}{ PCA-H $(n=134)$} & \multirow[t]{2}{*}{$P$-value } \\
\hline & n (\%) & Median (IQR) & n (\%) & Median (IQR) & \\
\hline \multicolumn{6}{|l|}{ Tumor number } \\
\hline Solitary & $371(75.6)$ & & $120(24.4)$ & & 0.383 \\
\hline Multiple & $57(80.3)$ & & $14(19.7)$ & & \\
\hline Tumor's size $(\mathrm{cm})$ & & $3.20(2.00,5.00)$ & & $3.05(2.04,4.60)$ & 0.522 \\
\hline \multicolumn{6}{|l|}{ Cut margin } \\
\hline Free & $407(76.5)$ & & $125(23.5)$ & & 0.416 \\
\hline Infiltrated & $21(70.0)$ & & $9(30.0)$ & & \\
\hline \multicolumn{6}{|l|}{ Differentiation } \\
\hline Well or moderate & $323(77.3)$ & & $95(22.7)$ & & 0.142 \\
\hline Poor & $100(72.5)$ & & $38(27.5)$ & & \\
\hline \multicolumn{6}{|l|}{ Vascular invasion } \\
\hline No & $386(76.9)$ & & $116(23.1)$ & & 0.236 \\
\hline Yes & $42(70.0)$ & & $18(30.0)$ & & \\
\hline \multicolumn{6}{|c|}{ lymph nodes infiltration } \\
\hline No & $342(76.7)$ & & $104(23.3)$ & & 0.567 \\
\hline Yes & $86(74.1)$ & & $30(25.9)$ & & \\
\hline
\end{tabular}

${ }^{*} P$ value $<0.05$ statistically significant; Mann-Whitney $\mathrm{U}$ test; Chi-square test; $\mathrm{n}(\%)=$ number (percentage).

PCA-H High PCA sufentanil dosage, PCA-NH Non high PCA sufentanil dosage

correlation analyses of sufentanil consumption $48 \mathrm{~h}$ after hepatectomy. These variables included age, age group ( $\leq 60$ and $>60$ years), operation time, operation time group ( $\leq 300$ and $>300 \mathrm{~min}$ ), extent of resection (minor hepatic resection and major hepatic resection), surgical approach (laparoscope and open), blood loss, intraoperative transfusion (yes/no), PONV (yes/no), preoperative albumin, and postoperative BUN. In this study, the above variables were included in the binary logistic regression analysis to evaluate their influence 
Table 5 Univariate analysis of influence on sufentanil consumption 48 hours after hepatectomy

\begin{tabular}{|c|c|c|c|}
\hline Characteristics & Number (n, \%) & Median, IQR & $P$ \\
\hline \multicolumn{4}{|l|}{ Age (years old) } \\
\hline$\leq 60$ & $308(54.8 \%)$ & $98.00(70.00,143.50)$ & \\
\hline$>60$ & $254(45.2 \%)$ & $82.00(62.75,120.00)$ & $<0.001^{*}$ \\
\hline \multicolumn{4}{|l|}{ Gender } \\
\hline Male & $316(56.2 \%)$ & $94.50(65.25,139.00)$ & \\
\hline Female & $246(43.8 \%)$ & $88.00(63.75,124.00)$ & 0.183 \\
\hline \multicolumn{4}{|l|}{ BMI $\left(\mathrm{kg} / \mathrm{m}^{2}\right)$} \\
\hline$\leq 25$ & $413(73.5 \%)$ & $90.00(65.50,131.50)$ & \\
\hline$>25$ & $149(26.5 \%)$ & $92.00(64.00,134.00)$ & 0.971 \\
\hline \multicolumn{4}{|c|}{ Intraoperative blood loss (ml) } \\
\hline$\leq 800$ & $487(86.7 \%)$ & $90.00(64.00,131.00)$ & \\
\hline$>800$ & $75(13.3 \%)$ & $99.00(73.00,155.00)$ & 0.054 \\
\hline \multicolumn{4}{|c|}{ Operative time (min) } \\
\hline$\leq 300$ & $422(75.1 \%)$ & $86.50(63.00,130.00)$ & \\
\hline$>300$ & $140(24.9 \%)$ & $103.0(71.25,140.00)$ & $0.008^{*}$ \\
\hline \multicolumn{4}{|c|}{ Intraoperative transfusion } \\
\hline No & $364(64.8 \%)$ & $86.50(63.00,125.00)$ & \\
\hline Yes & $198(35.2 \%)$ & $100.50(71.50,141.25)$ & $0.002^{*}$ \\
\hline \multicolumn{4}{|c|}{ Intraoperative transfusion of RBC (u) } \\
\hline$\leq 2$ & $453(80.6 \%)$ & $90.00(64.00,132.00)$ & \\
\hline$>2$ & $109(19.4 \%)$ & $94.00(72.50,139.00)$ & 0.165 \\
\hline \multicolumn{4}{|c|}{ Extent of resection } \\
\hline $\begin{array}{l}\text { Minor hepatic } \\
\text { resection }\end{array}$ & $272(48.4 \%)$ & $77.00(57.00,102.00)$ & \\
\hline $\begin{array}{l}\text { Major hepatic } \\
\text { resection }\end{array}$ & $290(51.6 \%)$ & $114.00(76.00,155.00)$ & $<0.001^{*}$ \\
\hline \multicolumn{4}{|l|}{ Surgical approach } \\
\hline Laparoscope & $302(53.7 \%)$ & $82.00(58.75,119.25)$ & \\
\hline Open & $260(46.3 \%)$ & $106.50(74.00,147.75)$ & $<0.001^{*}$ \\
\hline \multicolumn{4}{|l|}{ ASA classification } \\
\hline $1+11$ & $506(90.0 \%)$ & $91.25(64.88,133.00)$ & \\
\hline III & $56(10.0 \%)$ & $86.00(63.25,135.00)$ & 0.404 \\
\hline \multicolumn{4}{|c|}{ Introperative dexmedetomidine } \\
\hline No & $67(11.9 \%)$ & $91.50(65.00,133.00)$ & \\
\hline Yes & $495(88.1 \%)$ & $88.00(62.00,133.00)$ & 0.758 \\
\hline \multicolumn{4}{|c|}{ Postoperative NSAIDs } \\
\hline No & $170(30.3 \%)$ & $92.00(64.13,132.00)$ & \\
\hline Yes & $392(69.7 \%)$ & $87.50(64.00,133.88)$ & 0.848 \\
\hline \multicolumn{4}{|l|}{ PONV } \\
\hline No & $525(93.4 \%)$ & $90.00(64.00,130.50)$ & \\
\hline Yes & $37(6.6 \%)$ & $111.00(76.50,145.50)$ & $0.030^{*}$ \\
\hline \multicolumn{4}{|l|}{ Tumor number } \\
\hline Solitary & $491(87.4 \%)$ & $92.00(64.00,133.00)$ & \\
\hline Multiple & $71(12.6 \%)$ & $96.50(69.75,141.25)$ & 0.631 \\
\hline \multicolumn{4}{|l|}{ Tumor's size $(\mathrm{cm})$} \\
\hline$\leq 5$ & $408(72.6 \%)$ & $92.00(65.25,133.75)$ & \\
\hline$>5$ & $154(27.4 \%)$ & $86.50(63.00,127.00)$ & 0.491 \\
\hline \multicolumn{4}{|l|}{ Cut margin } \\
\hline Free & $532(94.7 \%)$ & $90.00(64.25,132.00)$ & \\
\hline
\end{tabular}

Table 5 (continued)

\begin{tabular}{|c|c|c|c|}
\hline Characteristics & Number (n, \%) & Median, IQR & $P$ \\
\hline Infiltrated & $30(5.3 \%)$ & $100.00(63.75,138.75)$ & 0.499 \\
\hline \multicolumn{4}{|l|}{ Differentiation } \\
\hline Well or moderate & $424(75.4 \%)$ & $90.00(64.00,130.00)$ & \\
\hline Poor & $138(24.6 \%)$ & $92.00(66.00,140.00)$ & 0.964 \\
\hline \multicolumn{4}{|l|}{ Vascular invasion } \\
\hline No & $502(89.3 \%)$ & $90.50(64.00,131.25)$ & \\
\hline Yes & $60(10.7 \%)$ & $94.50(65.75,146.50)$ & 0.438 \\
\hline \multicolumn{4}{|c|}{ lymph nodes infiltration } \\
\hline No & $446(79.4 \%)$ & $74.00(56.50,112.00)$ & \\
\hline Yes & $116(20.6 \%)$ & $97.00(64.75,134.75)$ & 0.540 \\
\hline \multicolumn{4}{|c|}{ Preoperative laboratory data } \\
\hline \multicolumn{4}{|c|}{ AST $(U / I)$} \\
\hline$\leq 40$ & $376(66.9 \%)$ & $93.50(64.00,135.50)$ & \\
\hline$>40$ & $186(33.1 \%)$ & $90.00(65.75,128.25)$ & 0.355 \\
\hline \multicolumn{4}{|l|}{$\operatorname{ALT}(\mathrm{U} / \mathrm{I})$} \\
\hline$\leq 40$ & $409(72.8 \%)$ & $94.00(64.00,134.50)$ & \\
\hline$>40$ & $153(27.2 \%)$ & $85.00(65.50,125.00)$ & 0.135 \\
\hline \multicolumn{4}{|c|}{ Alkaline phosphatase (U/l) } \\
\hline$\leq 94$ & $211(37.5 \%)$ & $94.00(62.00,134.00)$ & \\
\hline$>94$ & $351(62.5 \%)$ & $90.00(67.00,132.00)$ & 0.790 \\
\hline \multicolumn{4}{|l|}{ Albumin (g/l) } \\
\hline$\leq 35$ & $155(27.6 \%)$ & $83.00(63.00,125.00)$ & \\
\hline$>35$ & $407(72.4 \%)$ & $94.00(65.00,134.00)$ & 0.112 \\
\hline \multicolumn{4}{|l|}{ BUN (mmol/l) } \\
\hline$\leq 7.5$ & $522(92.9 \%)$ & $90.00(64.00,130.00)$ & \\
\hline$>7.5$ & $40(7.1 \%)$ & $111.50(72.00,151.75)$ & 0.072 \\
\hline \multicolumn{4}{|l|}{ Creatinine $(\mu \mathrm{mol} / \mathrm{l})$} \\
\hline$\leq 73$ & $362(64.4 \%)$ & $88.50(65.00,129.75)$ & \\
\hline$>73$ & $200(35.6 \%)$ & $95.00(64.00,139.00)$ & 0.218 \\
\hline \multicolumn{4}{|c|}{ Platelet count $\left(\mathbf{x} 10^{9} / 1\right)$} \\
\hline$\leq 100$ & $77(13.7 \%)$ & $97.00(67.00,139.00)$ & \\
\hline$>100$ & $485(86.3 \%)$ & $90.00(64.00,131.50)$ & 0.249 \\
\hline \multicolumn{4}{|c|}{ Prothrombin time (s) } \\
\hline$\leq 14$ & $444(79.0 \%)$ & $90.50(64.00,130.00)$ & \\
\hline$>14$ & $118(21.0 \%)$ & $93.50(64.75,137.00)$ & 0.515 \\
\hline \multicolumn{4}{|c|}{ Postoperative laboratory data } \\
\hline \multicolumn{4}{|c|}{ AST (U/l) } \\
\hline$\leq 40$ & $121(21.5 \%)$ & $98.00(70.00,143.00)$ & \\
\hline$>40$ & $441(78.5 \%)$ & $89.00(64.00,130.00)$ & 0.162 \\
\hline \multicolumn{4}{|l|}{$\mathrm{ALT}(\mathrm{U} / \mathrm{l})$} \\
\hline$\leq 40$ & $159(28.3 \%)$ & $97.00(72.00,134.00)$ & \\
\hline$>40$ & $403(71.7 \%)$ & $88.00(63.00,132.00)$ & 0.119 \\
\hline \multicolumn{4}{|c|}{ Alkaline phosphatase (U/I) } \\
\hline$\leq 94$ & $252(44.8 \%)$ & $92.50(64.00,134.00)$ & \\
\hline$>94$ & $310(55.2 \%)$ & $90.00(66.00,132.00)$ & 0.775 \\
\hline \multicolumn{4}{|l|}{ Albumin (g/l) } \\
\hline$\leq 35$ & $389(69.2 \%)$ & $88.00(63.00,131.00)$ & \\
\hline$>35$ & $173(30.8 \%)$ & $97.00(70,00,133.50)$ & 0.093 \\
\hline
\end{tabular}


Table 5 (continued)

\begin{tabular}{clll}
\hline Characteristics & Number (n, \%) & Median, IQR & $P$ \\
\hline BUN (mmol/l) & & & \\
$\quad \leq 7.5$ & $536(95.4 \%)$ & $90.00(64.00,132.00)$ & \\
$\quad>7.5$ & $26(4.6 \%)$ & $118.50(74.75,177.25)$ & 0.051 \\
Creatinine $(\mu \mathrm{mol} / \mathrm{l})$ & & & \\
$\quad \leq 73$ & $362(64.4 \%)$ & $90.00(63.00,133.00)$ & \\
$>73$ & $200(35.6 \%)$ & $95.00(68.25,132.75)$ & 0.310 \\
\hline
\end{tabular}

${ }^{*} P$ value $<0.05$ statistically significant; $P O N V$ Postoperative nausea and vomiting, BUN Blood urea nitrogen, ALT Alanine transaminase, AST glutamic oxalacetic transaminase.

on sufentanil consumption at $48 \mathrm{~h}$ after surgery. The obtained logistic model showed statistical significance $\left(\chi^{2}=111.656, P<0.001\right)$. Four variables were identified by the model, i.e., operation time ( $\mathrm{min})$, age group ( $\leq 60$ and $>60$ years), extent of resection (minor hepatic resection and major hepatic resection) and surgical approach (laparoscope and open). The specific parameter results are shown in Table 7. It can be concluded that a longer operation, age $\leq 60$ years, major hepatic resection and an open approach are risk factors for increased sufentanil consumption $48 \mathrm{~h}$ after hepatectomy.

\section{Discussion}

There have been many studies on the risk factors for long-term postoperative opioid use [11-13], but the factors influencing short-term postoperative opioid consumption have not been well studied. To more accurately determine the postoperative opioid needs of patients, we selected patients who used IV-PCA sufentanil at least $48 \mathrm{~h}$ after surgery as the subjects for the study. Other administration methods, such as oral administration or single intravenous injection, do not necessarily reflect patients' real demand for opioids after surgery.

We found that the dosage of opioids after hepatectomy varied considerably among individuals in APS ward rounds. It is generally believed that postoperative pain affects the use of opioids [14], but there are many influencing factors that deserve further exploration. In this study, we found that an age $\leq 60$ years, major hepatic resection, an open approach and a prolonged operation were likely to increase sufentanil consumption $48 \mathrm{~h}$ after hepatectomy. Understanding the risk factors for postoperative opioid use will help identify high-risk patients early, allowing us to make the necessary interventions to manage postoperative pain effectively. For high-risk patients, we should conduct close postoperative pain monitoring and increase the use of opioids, or we should appropriately reduce the use of opioids after surgery to avoid wasting these drugs.

In this study, we found that the dosage of sufentanil $48 \mathrm{~h}$ after hepatectomy was related to age. The use of opioids after surgery was higher in younger patients than in those who were older. This finding is consistent with the results of most previous studies [15-17]. Glasson et al. found that patients aged 54 years or less were more likely to use high-dose opioid analgesics than patients aged 55 or greater [15]. Similarly, Yen et al. and Lin et al. found that patients younger than 60 years received higher doses of opioid analgesics than those aged over $60[16,17]$.

Table 6 Correlation analysis of PCA sufentanil consumption in the first $48 \mathrm{~h}$ after surgery

\begin{tabular}{llllllllll}
\hline & Age & Operation time & Blood loss & RBC & FFP & Move pain POD1 & Move pain POD2 & Pre Albumin & Post BUN \\
\hline Sufentanil consumption & $r$ & -0.192 & 0.166 & 0.185 & 0.086 & 0.106 & 0.099 & 0.094 & 0.100 \\
& $P$ & $<0.001$ & $<0.001$ & $<0.001$ & 0.041 & 0.012 & 0.019 & 0.025 & 0.084 \\
& & & & & & & & &
\end{tabular}

Pre albumin Preoperative albumin, post BUN Postoperative blood urea nitrogen, POD1 Postoperative first day, POD2 Postoperative second day

Table 7 Multivariate logistic regression analysis of risk factors for high PCA sufentanil dosage

\begin{tabular}{|c|c|c|c|c|c|c|}
\hline & \multirow[t]{2}{*}{ B } & \multirow[t]{2}{*}{ SE } & \multirow[t]{2}{*}{$P$-Value } & \multirow[t]{2}{*}{ Odds ratio } & \multicolumn{2}{|c|}{$95 \% \mathrm{Cl}$ for $\operatorname{EXP}(\mathrm{B})$} \\
\hline & & & & & Lower & Upper \\
\hline Operative time (min) & 0.002 & 0.001 & 0.033 & 1.002 & 1.000 & 1.004 \\
\hline Age (group) & 0.866 & 0.233 & $<0.001$ & 2.377 & 1.505 & 3.755 \\
\hline Extent of resection & 3.981 & 0.542 & $<0.001$ & 53.587 & 18.527 & 154.988 \\
\hline Surgical approach & 2.516 & 0.518 & $<0.001$ & 12.381 & 4.488 & 34.157 \\
\hline Constant & -9.211 & 1.210 & $<0.001$ & $<0.001$ & & \\
\hline
\end{tabular}

$B$ Regression coefficient, SE Standard error, Cl Confidence interval, Age (group) Two age groups ( $\leq 60$ and $>60$ years old) 
How age affects postoperative opioid dosage is mainly considered as follows: First, older patients are more sensitive to opioid analgesics than younger patients [18]. Second, pharmacokinetic changes with age, including decreased volume of distribution, decreased metabolic function, and decreased elimination rate $[19,20]$, result in increased accumulation of opioids in elderly patients and prolonged effect intensity and duration. In addition, Kulkarni et al. [21] believe that young patients may experience greater emotional distress in the context of diseases that require surgery. Compared with young patients, elderly patients may regard serious diseases as the expected pain of aging. Such emotional upheavals, accompanied by symptoms of anxiety and depression, may exacerbate the severity of pain or disrupt healthy behaviors that may reduce pain symptoms. These emotional factors can also have a significant impact on postoperative pain. In general, the correlation between age and postoperative opioid use is generally recognized.

In this study, we found that the surgical approach was an influential factor for postoperative sufentanil dosage, suggesting a reduction in opioid dosage after laparoscopic surgery, which is consistent with the conclusions of many previous studies [22-24]. Mala $\mathrm{T}$ et al. found that patients undergoing laparoscopic hepatectomy used opioids for an average of 1 day, while patients undergoing open surgery used opiates for an average of 5 days [22].

A low demand for opioid treatment after laparoscopic surgery is related to a low degree of postoperative pain stress [25]. Since the incisions made in laparoscopic hepatectomy are small and only 4-5 holes need to be established, there is less pain stimulation [26-29]. Moreover, the local incision infiltration analgesia method can significantly reduce incisional pain and substantially reduce opioid use after surgery [30].

Additionally, the use of ultrasonic dissection in laparoscopic surgery significantly reduces smoke and eschar formation, fully ensuring that the surgeon has a clear visual field, and ultrasonic dissection does not damage the surrounding healthy tissues [31, 32]. Second, a harmonic scalpel, which is used to free the intrahepatic bile duct and blood vessels during laparoscopic surgery, can use electrocoagulation or clamping according to the thickness of the vessel for greater efficiency. Compared with tools in traditional open surgery, the use of a harmonic scalpel avoids the frequent replacement of other hand instruments and, at the same time, better guarantees electrocoagulation and hemostasis [31,32]. These factors can significantly reduce pain stress and the need for opioids after surgery.

Studies have found that the extent of resection is an independent influencing factor for postoperative pain. Major hepatectomy is very traumatic, and most of it is performed through laparotomy [33]. During the operation, it is often necessary to use a liver retractor to pull the ribs, and sometimes it is necessary to remove the xiphoid process to fully expose the lesion [34]. Postoperative drainage tubes often lead to severe postoperative pain in patients. This is the main reason for the increased consumption of sufentanil after surgery.

This study found that the operation time was a risk factor for a high postoperative sufentanil dosage. Patients with operation times longer than $300 \mathrm{~min}$ consumed more sufentanil within $48 \mathrm{~h}$ after the operation, but few related studies have investigated this factor. Loriga B et al. [35] found that the operation time was a significant risk factor for postoperative pain after vitreoretinal surgery, and this conclusion was related to the choice of anesthesia. Local anesthesia can enhance this correlation because with increases in the operation time and decreases in the anesthetic effect, postoperative pain stimulation increases. Silins V et al. [36] found that the operation time was an independent predictor for increased morphine consumption in children three days after the operation. It is believed that the operation time might be related to invasiveness, reflecting the degree of activity. Indeed, the liver is rich in blood vessels and has a complex anatomy, and the long operation could reflect the degree of trauma. The longer the operation, the stronger the inflammatory response caused by the inflammatory factors released from the injury, the higher the pain stress, and the greater the demand for opioid drugs after the operation. Unfortunately, the perioperative levels of leukocytes and other inflammatory indices were not included in this study, which could have helped determine the relationship between the duration of surgery and postoperative sufentanil dosage.

Opioid metabolism and clearance are affected by liver and renal function, but in this study, no relationship between perioperative liver and renal function and postoperative sufentanil dosage was found. Sufentanil is metabolized to normethyl sufentanil in liver microsomes, and the activity of normethyl sufentanil is only $10 \%$ that of sufentanil [37]. Renal function has a weak effect on the pharmacokinetics of sufentanil [38], which may be the main reason for these findings; of course, this requires further studies with large samples and multiple centers.

Sex has not been identified in previous studies as an influential factor for postoperative analgesic dosage consistency [39]. Many research studies have reported that women are more likely than men to suffer postoperative pain and use more analgesic drugs [40, 41]. Zheng $\mathrm{H}$ et al. found that women used $25.8 \%$ more opioids than men within $24 \mathrm{~h}$ after surgery [41]. It is generally concluded in many studies that women tend to report higher levels of anxiety and to exhibit factors associated with pain [40, 
41]. However, the current study is consistent with that conducted by Lin et al., in that no effect of sex on postoperative sufentanil consumption was found [17].

Similar to the study by Lee Y et al., the present study did not find that BMI was a risk factor for sufentanil dosage $48 \mathrm{~h}$ after surgery [14]. However, studies have shown that BMI is positively correlated with opioid consumption, but the etiology is still unclear [42, 43]. Kvarda $\mathrm{P}$ et al. considered that this finding might be related to the bias of surgeons against the use of opioids in obese patients because surgeons believed that patients with higher BMIs metabolized opioids differently and therefore used more analgesics in obese patients [42]. Previous studies on the relationship between these factors and postoperative opioid dosage are few, and further prospective studies are needed.

Similarly, this study did not find that PONV was a factor influencing the postoperative sufentanil dosage, although the postoperative sufentanil dosage was higher in the PONV group. The incidence of nausea and vomiting, in addition to opioid use, would also be affected by many factors, including the type of patient, surgery, and anesthesia [44, 45].

A recent meta-analysis of randomized controlled trials showed that dexmedetomidine saves opioids and can significantly reduce their dosage [46]. Studies have demonstrated that anti-inflammatory and analgesic drugs (e.g., NSAIDs), such as parexib or flurbiprofen, can reduce postoperative pain and reduce postoperative opioid use $[47,48]$. However, no effect of intraoperative dexmedetomidine and postoperative NSAID use on the postoperative sufentanil dosage was found in this study. In our study, we also did not find an association between intraoperative blood loss, preoperative platelet count, prothrombin time, or tumor factors and the 48-h postoperative sufentanil dose. There have been few studies on the relationship between these factors and postoperative opioid dosage, and further prospective studies are needed.

This study has several limitations. The design and outcome reliability of retrospective studies is inherently dependent on the accuracy and completeness of the documents available in electronic medical records and surgical reports. The present sample included 562 patients after hepatectomy. As a result, the sample size may not be sufficient to identify the effects of individual variables. Therefore, it is suggested that a larger sample size be studied in the future. In addition, this was a retrospective study that did not confirm the difference between the doses of different opioid analgesics. Future studies should include treatment with other opioids. Postoperative pain and opioid consumption are also influenced by a patient's psychological factors, the preoperative experience of pain, and the preoperative use of opioids [49-51]. It is difficult to evaluate these factors accurately in retrospective investigations; therefore, to comprehensively assess the factors affecting postoperative opioid dosage, it is necessary to add these predictive factors to future prospective studies.

Finally, patient education level and cognitive ability, postoperative infection, postoperative rehabilitation exercise, and other factors may be related to acute postoperative pain and opioid requirements, which can be further explored by adding corresponding observational indicators in future studies.

\section{Conclusions}

This retrospective study was designed to determine the influencing factors of sufentanil consumption $48 \mathrm{~h}$ after hepatectomy. The results of this investigation can help anesthesiologists improve the quality of perioperative pain and opioid management by considering these influencing factors. The findings of this study suggest that the factors influencing sufentanil consumption in liver cancer patients after hepatectomy are the age group $(\leq 60$ and $>60$ years), extent of resection (minor hepatic resection and major hepatic resection), surgical approach (laparoscope and open) and operation time (min). Patients younger than 60 years, patients who underwent major hepatic resection, patients who underwent surgery with an open approach and patients who had a longer operation tended to consume more sufentanil after surgery. These findings have a certain value for guiding the management of pain during the perioperative period in liver resection.

\section{Abbreviations}

BMI: Body mass index; ASA: American Society of Anesthesiologists; PCA: Patient-controlled analgesia; IV-PCA: Intravenous patient-controlled analgesia; NRS: Numerical rating scale; APS: Acute Pain Service Team; DEX: Dexmedetomidine; NSAIDs: Anti-inflammatory and analgesic drugs; POD: Postoperative day; RBC: Red blood cells; FFP: Fresh frozen plasma; PONV: Postoperative nausea and vomiting; BUN: Blood urea nitrogen; ALT: Alanine transaminase; AST: Glutamic oxalacetic transaminase.

\section{Acknowledgments}

We would like to thank the Department of Information, Sir Run Run Shaw Hospital, School of Medicine, Zhejang University for its support in obtaining case data. We further thank Dr. Xueyao Yin from the Department of Endocrinology, Sir Run Run Shaw Hospital, School of Medicine, Zhejang University for his support with the statistical analysis. We would also like to thank Dr. Tunan Yu from the General Surgery Department of Run Run Run Run Shaw Hospital for his suggestions on the research design. Finally, we would like to thank Dr. Xin Chen from Zhejiang University School of Medicine for her suggestions on the research writing.

\section{Authors' contributions}

YW and GC were responsible for the study design and organization. LT, MJL, SNQ, WBZ, SMT, and CYL gathered, analyzed and interpreted the data. YW and $C Y L$ were major contributors to writing the manuscript. All authors read and approved the final manuscript. 


\section{Funding}

The authors report was supported by Health and Family Planning Commission of Zhejiang Province (No. 2020375873). The funding agents play no role in the design of the study and collection, analysis, and interpretation of data and in writing the manuscript.

\section{Availability of data and materials}

The datasets used and/or analyzed during the current study are available from the corresponding author on reasonable request.

\section{Declarations}

\section{Ethics approval and consent to participate}

This single-center retrospective study was approved by the institutional review board (IRB) of Sir Run Run Shaw Hospital, School of Medicine, Zhejang University (IRB approval number: 20190725-030). Informed consent was waived because of the retrospective nature of this study.

\section{Consent for publication}

Not applicable.

\section{Competing interests}

The authors declare that they have no competing interests.

\section{Author details}

'Department of Anesthesiology, Sir Run Run Shaw Hospital, School of Medicine, Zhejang University, 3 Qingchun Road East, ShangCheng District, Hangzhou 310016 Zhejiang, People's Republic of China. ${ }^{2}$ Department of Pain, Affiliated Hospital of Jiangsu University, 438 Jiefang Road, Zhenjiang 212001, Jiangsu, People's Republic of China. ${ }^{3}$ Department of Nursing Education, Sir Run Run Shaw Hospital, School of Medicine, Zhejang University, 3 Qingchun Road East, ShangCheng District, Hangzhou 310016 Zhejiang, People's Republic of China.

\section{Received: 27 December 2020 Accepted: 23 November 2021} Published online: 07 December 2021

\section{References}

1. Lee $\mathrm{S}$, Whelan R. Immunologic and oncologic implications of laparoscopic surgery: what is the latest? Clin Colon Rectal Surg. 2006;19(1):005-12.

2. Gottschalk A, Sharma S, Ford J, Durieux ME, Tiouririne M. Review article: the role of the perioperative period in recurrence after cancer surgery. Anesth Analg. 2010;110(6):1636-43.

3. Neeman E, Ben-Eliyahu S. Surgery and stress promote cancer metastasis: new outlooks on perioperative mediating mechanisms and immune involvement. Brain Behav Immun. 2013;30:S32-40.

4. Sun HZ, Song YL, Wang XY. Effects of different anesthetic methods on cellular immune and neuroendocrine functions in patients with hepatocellular carcinoma before and after surgery. J Clin Lab Anal. 2016;30(6):1175-82.

5. Li H, Wei Y, Li B. Preoperative steroid administration in liver resection:a systematic review and meta-analysis. Hepatogastroenterology. 2013;60(121):160-9.

6. Olonisakin RP, Sotunmbi PT, Afuwape OO, Ayandipo O०, Adigun TAJAJMM. Regional anesthetic technique for laparoscopic appendicectomy in Ibadan. Afr J Med Med Sci. 2014;43(3):219-23.

7. Palmer PP, Miller RD. Current and developing methods of patient-controlled analgesia. Anesthesiol Clin. 2010;28(4):587-99.

8. Yang T, Zhang J, Lu JH, Yang GS, Wu MC, Yu WF. Risk factors influencing postoperative outcomes of major hepatic resection of hepatocellular carcinoma for patients with underlying liver diseases. World J Surg. 2011;35(9):2073-82.

9. Pang YY. The Brisbane 2000 terminology of liver anatomy and resections. HPB. 2000;2:333-9 HPB (Oxford) 2002; 4(2):99; author reply 99-100.

10. Chou R, Gordon DB, DE Leon-Casasola OA, Rosenberg JM, Bickler S, Brennan T, et al. Management of postoperative pain: a clinical practice guideline from the American pain society, the American Society of Regional Anesthesia and Pain Medicine, and the American Society of
Anesthesiologists' committee on regional anesthesia, executive committee, and administrative council. J Pain. 2016;17(2):131-57.

11. Sun EC, Darnall BD, Baker LC, Mackey S. Incidence of and risk factors for chronic opioid use among opioid-naive patients in the postoperative period. JAMA Intern Med. 2016;176(9):1286-93.

12. Sekhri S, Arora NS, Cottrell H, Baerg T, Duncan A, Hu HM, et al. Probability of opioid prescription refilling after surgery: does initial prescription dose matter? Ann Surg. 2018;268(2):271-6.

13. Shah A, Hayes CJ, Martin BC. Characteristics of initial prescription episodes and likelihood of long-term opioid use-United States, 20062015. MMWR Morb Mortal Wkly Rep. 2017;66(10):265-9.

14. Lee Y, Kim K. Factors related to the consumption of patient-controlled postoperative analgesics in Korea: A retrospective study. Pain Manag Nurs. 2020;21(5):449-55.

15. Glasson JC, Sawyer WT, Lindley CM, Ginsberg B. Patient-specific factors affecting patient-controlled analgesia dosing. J Pain Palliative Care Pharmacother. 2002;16(2):5-21.

16. Yen CR, Tsou MY, Mandell MS, Chan CT, Chan KH, Chen TH, et al. An analysis of patient variables that influence intravenous patient-controlled analgesic use of morphine with quantile regression. Anesthesiology. 2010;112(3):688-95.

17. Lin SP, Chang KY, Tsou MY, Chen TH. Serial analgesic consumptions and predictors of intravenous patient-controlled analgesia with cluster analysis. Clin J Pain. 2016;32(6):488-94.

18. Thomazeau J, Rouquette A, Martinez V, Rabuel C, Prince N, Laplanche $J \mathrm{~L}$, et al. Acute pain factors predictive of postoperative pain and opioid requirement in multimodal analgesia following knee replacement. Eur J Pain. 2016;20(5):822-32.

19. Greenblatt DJ, Sellers EM, Shader RI. Drug therapy: drug disposition in old age. N Engl J Med. 1982;306(18):1081-8.

20. Samer CF, Lorenzini KI, Rollason V, Daali Y, Desmeules JA. Applications of CYP450 testing in the clinical setting. Mol Diagn Ther. 2013;17(3):165-84.

21. Kulkarni AR, Pusic AL, Hamill JB, Kim HM, Qi J, Wilkins EG, et al. Factors associated with acute postoperative pain following breast reconstruction. JPRAS Open. 2017;11:1-13.

22. Mala T, Edwin B, Gladhaug I, Fosse E, Søreide O, Bergan A, et al. A comparative study of the short-term outcome following open and laparoscopic liver resection of colorectal metastases. Surg Endosc. 2002;16(7):1059-63.

23. Endo Y, Ohta M, Sasaki A, Kai S, Eguchi H, Iwaki K, et al. A comparative study of the long-term outcomes after laparoscopy-assisted and open left lateral hepatectomy for hepatocellular carcinoma. Surg Laparosc Endosc Percutan Tech. 2009;19(5):e171-4.

24. Del Pino S, Fischer L, Nashan B, Li J. Reduced opioid-demand and fewer pulmonary complications after laparoscopic liver resection in the posterior segments. Dig Surg. 2020;37(2):129-34.

25. Siniscalchi A, Ercolani G, Tarozzi G, Gamberini L, Cipolat L, Pinna AD, et al. Laparoscopic versus open liver resection: differences in intraoperative and early postoperative outcome among cirrhotic patients with hepatocellular carcinoma-A retrospective observational study. HPB Surg. 2014;2014:871251.

26. Goh BKP, Syn N, Teo JY, Guo YX, Lee SY, Cheow PC, et al. Perioperative outcomes of laparoscopic repeat liver resection for recurrent HCC: comparison with open repeat liver resection for recurrent HCC and laparoscopic resection for primary HCC. World J Surg. 2019;43(3):878-85.

27. Tsai KY, Chen HA, Wang WY, Huang MT. Long-term and short-term surgical outcomes of laparoscopic versus open liver resection for hepatocellular carcinoma: might laparoscopic approach be better in early HCC? Surg Endosc. 2019;33(4):1131-9.

28. Otsuka Y, Kaneko H. Laparoscopic liver resection in the treatment of HCC with liver cirrhosis: would it provide superiority to conventional open hepatectomy? Hepatobil Surg Nutr. 2017;6(5):356-8.

29. Wang J. Innovations promote the development of minimally invasive surgery. Laparosc Endosc Robot Surg. 2019;2(3):74-6. https://doi.org/10. 1016/j.lers.2019.07.003.

30. Zhang H, Du G, Liu YF, Yang JH, A-Niu MG, Zhai XY, et al. Overlay of a sponge soaked with ropivacaine and multisite infiltration analgesia result in faster recovery after laparoscopic hepatectomy. World J Gastroenterol. 2019;25(34):5185-96. 
31. Gobardhan PD, Subar D, Gayet B. Laparoscopic liver surgery: an overview of the literature and experiences of a single center. Best Pract Res Clin Gastroenterol. 2014;28(1):111-21.

32. Buell JF, Gayet B, Han HS, Wakabayashi G, Kim KH, Belli G, et al. Evaluation of stapler hepatectomy during a laparoscopic liver resection. HPB. 2013;15(11):845-50.

33. Al Samaraee A, Rhind G, Saleh U, Bhattacharya V. Factors contributing to poor postoperative abdominal pain management in adult patients: a review. Surgeon. 2010;8(3):151-8.

34. Kalkman CJ, Visser K, Moen J, Bonsel GJ, Grobbee DE, Moons GMK. Preoperative prediction of severe postoperative pain. Pain. 2003;105(3):415-23.

35. Loriga B, Di Filippo A, Tofani L, Signorini P, Caporossi T, Barca F, et al. Postoperative pain after vitreo-retinal surgery is influenced by surgery duration and anesthesia conduction. Minerva Anestesiol. 2019;85(7):731-7.

36. Silins V, Brasher C, Antus F, Michelet D, Hilly J, Grace R, et al. Predicting postoperative morphine consumption in children. Anaesth Crit Care Pain Med. 2017;36(3):179-84

37. Zöllner C, Schäfer M. Opioids in anesthesia. Anesthesist. 2008;57(7):729-40.

38. Fyman PN, Reynolds JR, Moser F, Avitable M, Casthely PA, Butt K. Pharmacokinetics of sufentanil in patients undergoing renal transplantation. Can J Anaesth. 1988;35(3(Pt 1)):312-5.

39. Ip HY, Abrishami A, Peng PW, Wong J, Chung F. Predictors of postoperative pain and analgesic consumption: A qualitative systematic review. Anesthesiology. 2009;111(3):657-77.

40. Nandi M, Schreiber KL, Martel MO, Cornelius M, Campbell CM, Haythornthwaite $\mathrm{J}$, et al. Sex differences in negative affect and postoperative pain in patients undergoing total knee arthroplasty. Biol Sex Differ. 2019;10(1):23.

41. Zheng H, Schnabel A, Yahiaoui-Doktor M, Meissner W, Van Aken H, Zahn $P$, et al. Age and preoperative pain are major confounders for sex differences in postoperative pain outcome: A prospective database analysis. PLoS One. 2017;12(6):e0178659.

42. Kvarda P, Hagemeijer NC, Waryasz G, Guss D, DiGiovanni CW, Johnson AH. Opioid consumption rate following foot and ankle surgery. Foot Ankle Int. 2019;40(8):905-13.

43. Abrecht CR, Cornelius M, Wu A, Jamison RN, Janfaza D, Urman RD, et al. Prediction of pain and opioid utilization in the perioperative period in patients undergoing primary knee arthroplasty: psychophysical and psychosocial factors. Pain Med. 2019;20(1):161-71.

44. Gan TJ, Diemunsch P, Habib AS, Kovac A, Kranke P, Meyer TA, et al. Consensus guidelines for the management of postoperative nausea and vomiting. Anesth Analg. 2014;118(1):85-113.

45. Nathan N. Management of Postoperative Nausea and Vomiting: the 4th consensus guidelines. Anesth Analg. 2020;131(2):410

46. Blaudszun G, Lysakowski C, Elia N, Tramer MR. Effect of perioperative systemic a2 agonists on postoperative morphine consumption and pain intensity: systematic review and meta-analysis of randomized controlled trials. Anesthesiology. 2012;116(6):1312-22.

47. Rayakorn M, Adisorn T, Chininthon P, Nida P, Unchana K, Nipawan J, et al. Comparison of preoperative and postoperative Parecoxib Administration for Pain Control Following Major Spine Surgery. Asian Spine J. 2018;12(5):893-901.

48. Sun MY, Cong XH, Chang EQ, Miao MR, Zhang JQ. Efficacy of Flurbiprofen for postoperative pain in Chinese surgical patients: A meta-analysis. J Surg Res. 2020;252:80-8.

49. Hah JM, Bateman BT, Ratliff J, Curtin C, Sun E. Chronic opioid use after surgery: implications for perioperative Management in the Face of the opioid epidemic. Anesth Analg. 2017;125(5):1733-40.

50. Dindo L, Zimmerman MB, Hadlandsmyth K, Marie B, Embree J, Marchman $J$, et al. Acceptance and commitment therapy for prevention of chronic postsurgical pain and opioid use in at-risk veterans: A pilot randomized controlled study. J Pain. 2018;19(10):1211-21.

51. Nixon DC, Schafer KA, Cusworth B, McCormick JJ, Johnson JE, Klein SE. Preoperative anxiety effect on patient-reported outcomes following foot and ankle surgery. Foot Ankle Int. 2019;40(9):1007-11.

\section{Publisher's Note}

Springer Nature remains neutral with regard to jurisdictional claims in published maps and institutional affiliations.

Ready to submit your research? Choose BMC and benefit from:

- fast, convenient online submission

- thorough peer review by experienced researchers in your field

- rapid publication on acceptance

- support for research data, including large and complex data types

- gold Open Access which fosters wider collaboration and increased citations

- maximum visibility for your research: over $100 \mathrm{M}$ website views per year

At BMC, research is always in progress.

Learn more biomedcentral.com/submissions 